-Communication-

\title{
An Enhanced Anion Doping Process at NBR/Polypyrrole Composite Polymer Electrode
}

\author{
Katsuhiko NAOI and Tetsuya OSAKA*
}

Received July 4, 1986 ; Accepted July 31, 1986

\section{INTRODUCTION}

It has already been found that an electrochemical doping-undoping process at a polypyrrole(PPy) electrode is strongly affected by the film morphology ${ }^{9}$ ) and that a diffusion rate of anions within the film can be accelerated by choosing suitable conditions for film formation such as the formation potential 1,2$)$ and electrolyte anions ${ }^{3}$. Recently, a unique preparation of a PPy-based composite polymer ${ }^{5}$ has been attempted as one of the variations of electro-polymerization materials. As a new conducting material composed of PPy and an insulating material, we prepared polypyrrole on Pt substrate which has initially been coated with a nitrile rubber(NBR) insulating film. In the procedure of NBR/PPy film formation, the primarily coated NBR film may play a role of a host polymer which regulates a direction of the growth of the guest or the PPy film. In this communication, we report interesting behavior about a highly accelerated anion doping process at a Pt/NBR/PPy electrode.

\section{EXPERIMENTAL}

Reagent grade acetonitrile(AN) was used as a solvent without further purification. All the potentials were referred to the $\mathrm{Ag} / \mathrm{Ag}^{+}$electrode. Pt/PPy was formed by electro-oxidative polymerization at $0.8 \mathrm{~V}$ in $\mathrm{AN}$ solution containing $0.1 \mathrm{~mol}$ $\mathrm{dm}^{-3}$ tetra-n-buthyl ammonium perch lorate $\left(\mathrm{TnBAClO}_{4}\right)$ and $0.1 \mathrm{~mol} \mathrm{\textrm {dm } ^ { - 3 }}$ pyrrole monomer. For the preparation of $\mathrm{Pt} / \mathrm{NBR} / \mathrm{PPY}$, the Pt substrate was firstly coated with commercially available NBR prepolymer (ca.2 $\mu \mathrm{m}$ thick) and dried. Then PPy was growed through the NBR film in the same manner as in the case of Pt/PPy film. Film thickness was controlled by the passed charges. Cyclic voltammograms were obtained in 0.1 mol $\mathrm{dm}^{-3}$ $\mathrm{TnBAClO}_{4} / \mathrm{AN}$. Frequency dispersions of the electrode impedance were measured from $1.0 \mathrm{~Hz}$ to 2.5 $\mathrm{kHz}$ by using the FFT impedance method ${ }^{6)}$. Apparent diffusion coefficients ( $D_{a p p}$ ) were determined from the slope of cottrell plot obtained by the potential step chronoamperometry 4 ).

\section{RESULTS AND DISCUSSION}

Figure 1 shows typical cyclic voltamnograms (20-30th cycles) for the Pt/PPy and Pt/NBR/PPy electrodes with the same formation charges $(1.0$ $\left(\mathrm{cm}^{-2}\right)$. As can easily be seen, the anodic and cathodic currents for the Pt/NBR/PPy electrode show remakable increases. Therefore, the anion doping/undoping process at the Pt/NBR/PPY film is considered to be highly accelerated. It

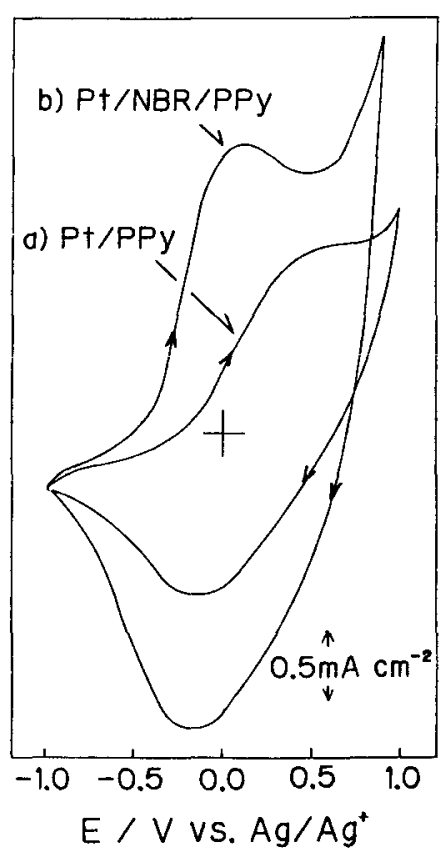

Fig.1 Cyclic voltammograms for a) $\mathrm{Pt} / \mathrm{PPy}$ and b) $\mathrm{Pt} / \mathrm{NBR} / \mathrm{PPy}$ film electrodes in $0.1 \mathrm{~mol} \mathrm{\textrm {dm } ^ { - 1 }}$ TnBACI0/AN at a scan rate of $10 \mathrm{mV} \mathrm{s} 41$. The thickness of NBR film was ca. $2 \mu \mathrm{m}$.

\footnotetext{
*Department of Applied Chemistry, School of Science and Engineering, Waseda University (3-4-1 Okubo,Shinjuku-ku,Tokyo,Japan)
} 
is also observed that the doping charge for the Pt/NBR/PPy electrode far outnumbers the Pt/PPy electrode. The behavior similar to that in Fig.1 was also observed for other Pt/NBR/PPy films of more charged PPy films $(2.0,5.0$ and $10 \mathrm{C}$ $\mathrm{cm}^{-2}$ ) formed on the same thickness of NBR films.

Electrode impedance for these electrodes was measured at each anodic peak potential and was demonstrated in Fig.2. The total impedance for the Pt/NBR/PPy electrode is obviously smaller than that for the Pt/PPy electrode. The reaction resistance for the oxidation process would be reflected in the diameters of these semi-circles. The doping process at the Pt/NBR/PPy electrode is considered to be easier than that of the Pt/PPy electrode. The $D_{a p p}$ values obtained for Pt/PPy and the $\mathrm{pt} / \mathrm{NBR} / \mathrm{PPy}$ electrodes of $1 \mathrm{C} \mathrm{cm}^{-2}$ charged films are $5.01 \times 10^{-11} \mathrm{~cm}^{2} \mathrm{~s}^{-1}$ and $1.04 \times 10^{-10} \mathrm{~cm}^{2} \mathrm{~s}^{-1}$, respectively. These values together with the results of Cole-cole plots can support well evidence of an remarkable improvement of the anion doping process at the Pt/NBR/PPy electrode.

Brief images of PPy film growth for Pt/PPy and Pt/NBR/PPy electrodes could be schematically illustrated in Fig.3. The illustrations would better explain the formation procedure of two electrodes. When PPy is formed on a bare Pt substrate, the resulting film will show a random growth. In contrast, for the Pt electrode initially coated with the NBR film, the electrode system is considered to be insulated at first. However, the AN/ $\mathrm{TnBAClO}_{4}$ solution gradually penetrates into NBR film, which forms fine channels through the NBR film at the same

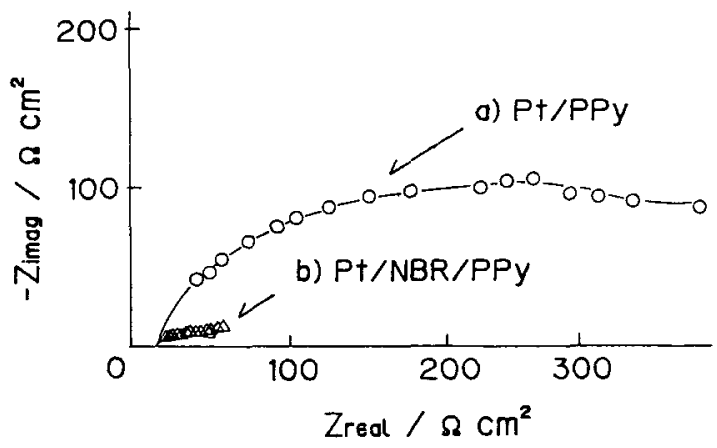

Fig.2 Typical Cole-Cole plots for a) Pt/PPy and b) Pt/NBR/PPy film electrodes in $0.1 \mathrm{~mol}$ $\mathrm{dm}^{-3} \mathrm{TnBAC} 10_{4} / \mathrm{AN}$, when the potentials are polarized at peak potentials a) $0.5 \mathrm{~V}$ and $\mathrm{b}$ ) $0.0 \mathrm{~V}$, respectively.

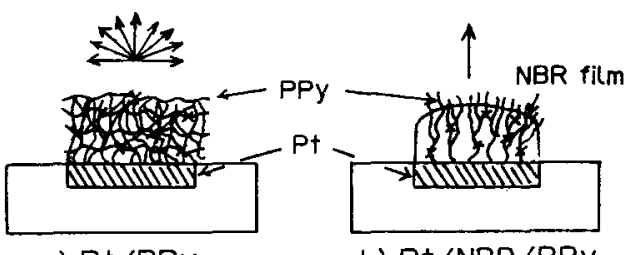

a) Pt/PPy

b) Pt/NBR/PPY

Fig. 3 Typical models of the film growth for a) Pt/PPy and b) Pt/NBR/PPy electrodes. The arrows represent the direction of $P P y$ film growth. time. The PPy film may grow along these channels. One of the most probable reasons for the improvement would be owing to an easier diffusion in the direction perpendicular to the substrate across such an oriented structure of the PPY film.

\section{REFERENCES}

1) K.okabayashi, F.Goto, K.Abe, T.Yoshida and M.Morimoto, Proceeding of $25 t h$ Battery Symposium in Japan, p.260(1984).

2) Y.Yatsuda, H.Sakai and T.Osaka, J.Chem.Soc.Jpn., 7,1331(1985).

3) H.Sakai, K.Naoi, T.Hirabayashi and T.Osaka, Denki Kagaku, 54,75(1985).

4) K.Naoi, H.Sakai, S.Ogano and T.Osaka, 3 rd Int. Meet. on Li Batteries, Extended Abs., p.266, Kyoto (1986); J.Power Sources, to be published.

5) O.Niwa and T.Tamamura, J.C.S.Chem.Commun., 1984,817.

6) T.Osaka and K.NaOi, Bull.Chem.Soc.Jpn., $\underline{5} \underline{5}, \overline{36(1982) .}$ 